\title{
On Sensor Technologies in CVD Diagnosis
}

\author{
Winncy Du
}

\begin{abstract}
Major applications of sensor technologies in cardiovascular disease (CVD) diagnoses include (1) detect heart pulse waveforms, (2) measure blood properties, (3) create heart or vessel images, and (4) establish wireless CVD detecting or monitoring network. Sensors such as piezoresistive and strain gauge sensors have been used to automatize or modernize TCPD; conductive, impedance, and optical sensors have been utilized to obtain ECG, ICG, and PPG waveforms, respectively, to assess the patient's cardiovascular status; piezoelectric sensors are used in ultrasound devices to generate a moving picture of the heart, show its size, shape, structure, and how well its valves are working; piezoelectric sensors, as well as electromagnetic and capacitive sensors can also be used to detect and record the sounds from the heart or blood flow in arteries and veins. Radiation sensors detect penetrated $X$-ray to create CCT images; inductive sensors (coils) can pick up $10-100 \mathrm{MHz}$ radio waves released from soft tissues after their H-atoms' magnetic resonance and create a cardiac MRI.
\end{abstract}

Index Terms-Cardiovascular disease, diagnosis, sensor, transducer.

\section{INTRODUCTION}

Sensors have been playing key roles in all aspects of health care, especially diagnosing Cardiovascular Disease (CVD) — the No.1 killer in the United States and the leading disease that took away 17.3 million lives worldwide in 2013 [1]. Unlike most of other diseases, some CVDs can cause sudden death, especially in people who physically appear heathy and fit. Therefore, reliable diagnosis methods are extremely important to uncover hidden or silent heart diseases to prevent sudden death. Moreover, the cost for detecting and treating CVDs is very high, thus early diagnosis and prevention post a significant financial benefit as well. Sensor technologies for heart diseases have attracted great attention from health care, engineering, and information processing societies and industry.

The cardiovascular disease (CVD) is a class of diseases that affect the heart or blood vessels. It includes dysrhythmias/arrhythmia (abnormalities of heart rhythm) cardiomyopathy (cardiac muscle disease, congenital heart defect (heart structure malformations existing at birth), myocarditis (inflammation of the muscular part of the heart), myocardial ischemia/myocardial infarction (heart attack), rheumatic heart disease (heart muscle or valve damage due to rheumatic fever), valvular heart disease (damage or defect in the valves), vascular disease (diseases of arteries, veins, and lymph vessels), and stroke (poor blood flow to the brain results in cell death). Major sensor technologies that are used to detect CVDs are discussed below.

Manuscript received June 2, 2015; revised October 13, 2015.

Winncy Du is with the Mechanical Engineering Department, San Jose State University, San Jose, CA 95192 USA (e-mail: winncy.du@sjsu.edu).

\section{Sensors for Traditional Chinese Pulse Diagnosis}

Traditional Chinese medicine (TCM) is a practice that has been used for thousands of years for diagnosing various cardiovascular diseases [2]. TCM suggests that the pulse condition is closely related to the pulsation of the heart and unobstructed blood vessels. Deficiency of blood circulation volume can make pulse appear thin; changes in blood viscosity can make pulse be slippery or astringent; capacity and state of blood, the thickness and the elasticity of vessel walls, also directly influence pulse patterns [3]. From a hemodynamic point of view, the heart beating creates the arterial pulsation, resulting in flow waves that propagate throughout the arterial system such as the coronary and cerebral arteries. Thus, the central pressure bears a stronger relationship to vascular damage and prognosis [4].

TCPD is based on the patterns of pulses felt by three fingers (index, middle, and ring fingers) placed over the radial artery at the wrist [5]. Three different pressures (light, medium, and high) are applied to the artery to reveal different responses. Based on the six characteristic qualities of the wrist pulse patterns-pulse width, depth, strength, rate, length, and rhythm —as many as 29 patterns can be distinguished and related to diagnosis of a wide range of diseases and conditions, ranging from heart diseases, liver malfunctions, and pregnancy [6], [7], to adult age estimation [8]. The diagnostic result of TCPD, however, depends highly on personal knowledge and experience of the physicians, which lacks standard, consistent, and automatic features. Current research aims at modernizing TCPD using sensor and information technologies. The wrist pulse waveforms can be detected by piezoresistive sensors, and then processed, compared (with the patterns stored in an expert data based library), and interpreted for disease diagnosis and health condition. Fig.1(a) shows a wearable automatic TCPD meter developed by Nair and Du [9]. The detected waves were amplified and then sent through a series of filters (see Fig.1(b)). This device provides current TCM practitioners with a quantitative and visual tool for pulsation diagnosis. It can also be used for general healthcare and self-health monitoring.

Luo et al. designed a bi-sensing pulse diagnosis instrument (BSPDI) using strain gauges and a pressure sensor array [10]. Three strain gauges (each for each finger) are used to measure the depth of each finger pushing onto the patient's wrist, while the pressure sensor array is used to obtain the wrist pulses. A wrist fixture is to ensure proper wrist position and orientation for accurate pulse measurement [11]. Perica et al. proposed a wrist-mounted "stopwatch" cardiac monitor that uses a pressure transducer to measure pulse rate on wrist (US Pat. No. 4, 256, 117). To advance TCM, Peng et al. developed a flexible capacitive tactile sensor array using 
commercial flexible printed circuit boards (FPCB) for real-time pulse measurement with CMOS readout circuits (see Fig. 2) [12]. Each FPCB consists of a polyimide insulation layer sandwiched between two copper layers. Two pieces of FPCBs were aligned and assembled to form the top and bottom sensing electrodes with a photoresist element as the spacer. The total thickness of the sensor layers is $13 \mu \mathrm{m}$. Capacitive sensing is chosen for its high sensitivity, ease of fabrication, and low power consumption. Wang and Xiang have showed that pathological changes have influences on the power density distribution of pulse signals [13]. Lau and Wang identified five and four pulse waveform contour prototypes for patients with chronic renal diseases and heart failure, respectively [6].

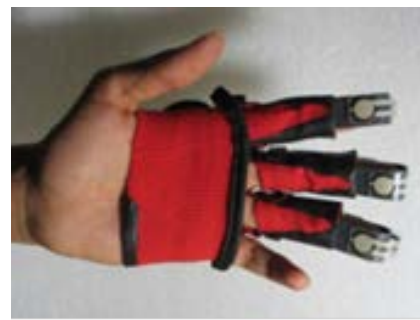

(a)

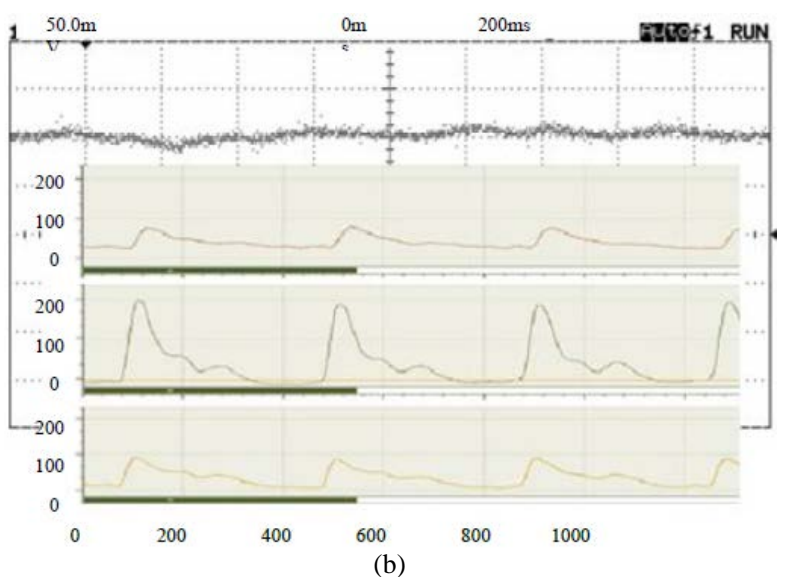

Fig. 1. A wearable pulse meter (a) and pulse waveforms detected (b).

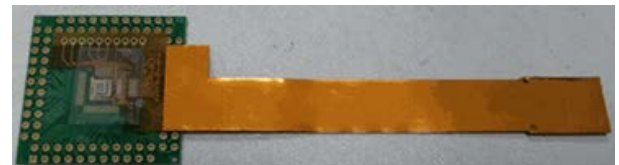

Fig. 2. A flexible capacitive tactile sensor array with a readout chip.

\section{SENSORS FOR ECG, ICG, AND PPG}

An electrocardiograph (ECG) detector detects and records the heart's electrical activity - the strength and timing of electrical signals as they pass through the heart. An ECG provides fundamental information for heart state - heart rate, rhythm (steady or irregular), heart rate variability (HRV), and cardiac cycle (P-QRS-T). Based on an ECG, a doctor can diagnose many cardiac disorders such as bradycardia, tachycardia, hypertrophy and apnea. For instance, in a healthy heart, the ECG's baseline is equivalent to the isoelectric line $(0 \mathrm{mV})$; while in a diseased heart the baseline may be elevated (cardiac ischemia) or depressed (myocardial infarction) relative to the isoelectric line.

The primary factors that affect accurate ECG sensing are: position, orientation, number and type of electrodes; electrode contact noise; movement artifacts; baseline drift; power-line/electromagnetic interference. To obtain high quality ECGs, advanced ECG devices are equipped with shielding and signal conditioning circuits [14], gel-free electrodes, contactless electrodes (garments with embedded electrodes) [15], or other sensors (e.g., a 3D accelerometer to measure the patient's motion, and use this feedback to cancel out motion artifacts). Different from traditional conductive ECG devices that use electrodes, the latest capacitive ECG devices utilize the capacitive coupling principle to obtain ECG signals, which eliminates the electrochemical half-cell potential that may vary with ion concentrations on the skin. Lo et al. developed a wristwatch ECG monitor, thus the user can walk and jog while taking ECG measurement (U.S. Pat. 5,738,104 and 5,876,350). The circuit inside the system can filter out muscle motion noise effectively.

Impedance Cardiogram (ICG) is obtained using a thoracic impedance method of measuring thorax fluid levels to assess the cardiovascular status of a patient. During the measurement, an alternating (electrical) current (AC) passes through the thorax. With each cardiac cycle, the thoracic fluid levels change, causing the impedance change. A typical ICG has a low-voltage (2.5-4.0 mV) and high-frequency (70-100 kHz), and a waveform as shown in Fig. 3 [16]. ICG is used to noninvasively track blood volume changes that occur during the cardiac cycle. It is a cost-effective approach that has been approved to provide reliable hemodynamic values to quantify the severity of heart failure. ICG is very useful for diagnostic evaluation, longitudinal prognostication, and therapeutic decisions. Ambulatory ICG monitoring allows the estimation of cardiac hemodynamic parameters (such as stroke volume or cardiac output) during transient events. This technique is a useful tool to facilitate more convenient and safer treatment plans for cardiovascular patients.

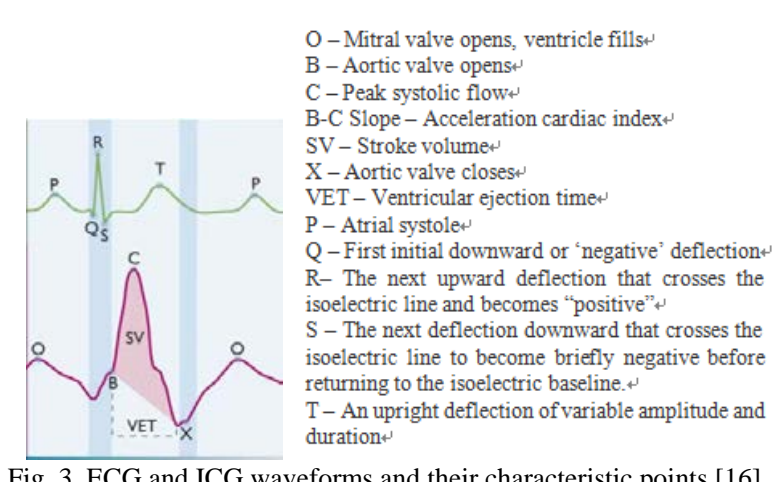

Fig. 3. ECG and ICG waveforms and their characteristic points [16].

ECG indicates the electric function of the heart, while ICG indicates the mechanical function of the heart. To monitor the electric and mechanical functions of the heart simultaneously, a multifunction wearable integrated sensor was developed which can detect ECG, ICG, HRV, and physical activity simultaneously and continuously [17].

A plethysmogram (PPG) test is performed by placing blood pressure cuffs on the extremities (e.g., fingers) to measure the systolic pressure and then display each pulse wave. With each cardiac cycle the heart pumps blood to the periphery and reaches the skin as well as the subcutaneous tissue. The test compares the systolic blood pressure of the 
lower extremity to the upper extremity, to help rule out disease that blocks the arteries in the extremities. Most PPG devices use optical methods to obtain plethysmograms. A light-emitting diode (LED) illuminates the skin on the finger (e.g., a pulse oximeter as shown in Fig.4), and the amount of light either reflected or transmitted through the finger is measured by a photodiode. Thus the pulsatile changes of blood volume in tissue are converted to changes in light reflection or absorption [18]. The LED used is in the range of $600 \mathrm{~nm}$ to $1400 \mathrm{~nm}$. A newly developed optical sensor system uses three wavelengths for measuring oxygenation and pulse [19]. PPG is mainly used for measuring pulsation in a capillary network. Because blood flow to the skin can be modulated by multiple other physiological systems, PPG can also be used to evaluate breathing, hypovolemia, and other circulatory conditions.
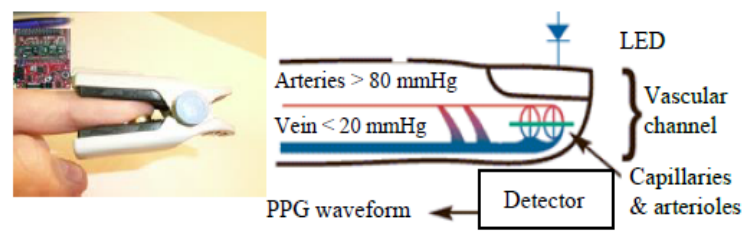

Fig. 4. A pulse oximeter and its working.

A major challenge faced by PPG devices is that the pulse is generally attenuated by the tissue between the artery and the photo detector. In addition, muscle movement may also create substantial noise at the sensors, making it difficult to identify blood pressure pulses reliably since the intensity of the transmitted or reflected light signal is significantly disturbed. Ambient lighting conditions also play an important role in the effectiveness of PPG technology. Other technologies that use strain gauges, piezoelectric films, infrared optical coupler pairs and fiber optic sensors so far, can only measure heart rate with reasonable reliability when the subject is still. They are not practical for sports, fitness and rehabilitation applications where the subject is moving.

\section{Ultrasound SENSORS FOR CVD DETECTION}

A typical ultrasonic sensor used in medical diagnosis utilizes a piezoelectric crystal to generate a sound wave (in a pulse mode, with an operating frequencies from $20 \mathrm{kHz}$ up to several gigahertz), and then pick up the reflected signal (echo) by the same crystal after the output power source is turned off. Some ultrasonic sensors use two piezoelectric elements: one as the transmitter, and the other as the receiver, which allows continuous detection. The time required to receive the echo depends upon the distance between the sensor and the object. The frequency shift (Doppler shift) is dependent upon the speed of the moving object. Thus, ultrasound sensors can create a moving picture of the heart; show its size, shape, structure, and how well its chambers and valves are working; detect the motion of a blood vessel wall (see Fig. 5) [20], [21]; provide an internal view of arteries; and extract pulse rate information (echocardiogram). Echo can also show the areas of poor blood flow to the heart, blood clots inside the heart, areas of the heart muscle that are not contracting normally, fluid buildup in the pericardium (the sac around the heart), problems with the aorta, and previous injury to the heart muscle caused by poor blood flow. Freeman et al. developed a cardiac wristwatch monitor for heart and pulse rate measurement using ultrasonic transducers that are encased in epoxy, covered with an insulated coating, and mounted in the wrist strap portion [22].

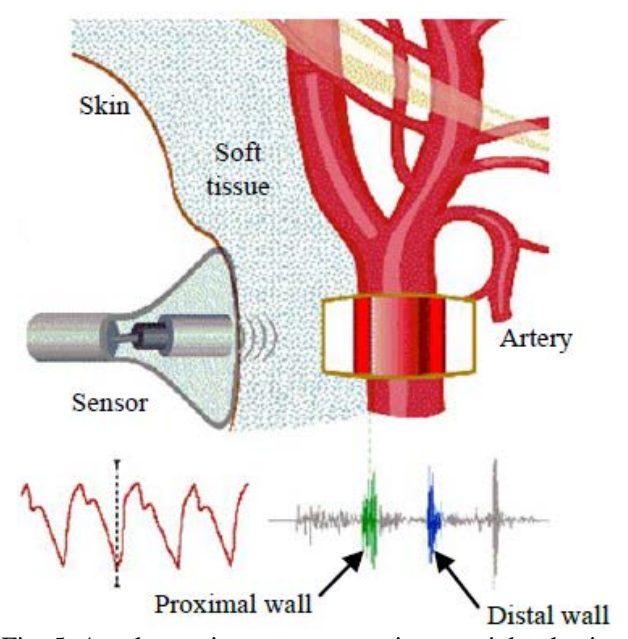

Fig. 5. An ultrasonic sensor measuring arterial pulsation.

One advantage of the ultrasonic technique is low cost and usually low power consumption especially with the latest piezoelectric materials and microelectronic development. Major challenges of this technique include its requiring high speed power switching due to the short distance between sensor and object, especially for superficial blood vessel detection. Muscle movement will also generate "echo" with the signal-to-noise-ratio near to 1 - making it difficult to distinguish the heart signal from muscle noise. However, the Doppler shift, due to blood flow is distinctly different from the shifts due to muscle artifacts or tissue movement (the shift due to blood flow is higher in frequency than that due to muscle motion), thus even though the muscle motion induced signals are larger in amplitude, they may still be filtered out by a high pass filter in either analog or digital form to retain the blood flow signals. In this respect, the ultrasound method is superior to infrared, pressure sensing, and even ECG based techniques. Another challenge is that ultrasonic waves are prone to diffraction and attenuation at the interface of two media of different densities. Any air gap at the interface or any air bubbles in the media will make ultrasonic sensing unreliable. Therefore, it has been a standard practice to apply water or an aqueous gel between the transducer and the subject to eliminate any air gaps. Water and gels, however, dry up quickly and it is not practical to apply water or gel frequently for continuous heart monitoring. One of methods to overcome this problem is to use an array of transducers to make firm contact without air gaps between the sensor and the subject [20]. However, this significantly increases the complexity and cost of the transducer device and its driving electronics, plus sometimes the air gap will not be completely removed due to body hairs or the variation of skin condition. Another solution is to use two sets of transducers (e.g., one located at the radial artery and the other at the ulnar artery) and let at least one of them reliably measure the Doppler shift, then two sensor signals will be used to eliminate the air gap effect in the final output signal [23]. 


\section{ACOUSTIC SENSORS FOR CVD DETECTION}

Different from an ultrasonic sensor that emits a sound wave and measure its echo, acoustic sensors detect and record the sounds and murmurs made by the heart or blood flow in arteries and veins, often called phonocardiograph (PCG). The commonly used stethoscope is based on the acoustic principle. With it, doctors can listen to the internal sounds from the lungs, hearts, and intestines. Placing a fetal stethoscope against the abdomen of a pregnant woman, a doctor can listen to the heart beating of the fetus.

One problem with conventional acoustic stethoscopes is that the sound level is extremely low, thus electronic stethoscopes are developed to amplify the body sounds electronically, where acoustic sound waves are converted to electrical signals to be amplified and processed. To avoid amplify body noise and contact artifacts as well, many analog or digital filtering techniques are integrated in the latest stethoscopes so that the heart sound signals, whose frequency content lies between about $100 \mathrm{~Hz}$ and $600 \mathrm{~Hz}$, is reserved while other noise signals above or below this range are attenuated. The transducers used to convert sound pressure to electrical voltage/current vary widely - ranging from piezoelectrical crystals (pressure change results in voltage change), to electromagnetic diaphragms (moving-coil induces electrical current in a magnetic field), and to variable capacitors (one moving plate causes the voltage potential between two places to change). $3 M$ company uses a piezo-electric element placed within foam behind a thick rubber-like diaphragm. Thinklabs uses a conductive inner surface to form a capacitive sensor. This preserves the acoustic sound from internal organs with the benefits of amplification. Besides audio output, an electronic stethoscope can also be made visual output and wireless device. Duff et al. designed an acoustic sensor array to detect coronary artery disease (CAD) based on heart sound data [24]. The sensor is a capacitive type with a 0.01 microfarad capacitance per element. An additional sensor is used as a "reference" to measure the ambient acoustical noise, so that the ambient acoustical noise is subtracted from the cardiac acoustical activity. The sensor array output is amplified and passed through a bandpass filter. This output is then compared to certain statistical characteristics of the waveform, yielding a final screening index (indicates the type of heart disease). This device, with a displayer, provides an apparatus by which non-medical personnel may screen patients for cardiovascular disease without the supervision of professional physicians.

\section{CARDIAC COMPUTERIZED TOMOGRAPHY (CT)}

Noninvasive imaging for detecting CHDs has evolved significantly over the last 50 years. Cardiac computerized tomography (CCT) scan uses an X-ray to obtain pictures of the heart and vessels. During the scan, an X-ray device rotates around the chest area (see Fig. 6) [25], and the X-ray penetrates the body. Based on the ability of various bodily structures to block the X-ray beam, the detectors on the other side of the body receive the penetrated X-ray data. Each $360^{\circ}$ rotation creates a slice - a $2 \mathrm{D}$ sectional image. When the machine moves axially, multiple slices are obtained. A computer then "packs" these 2D images together to create a 3D image of the whole heart. This allows both inside and outside of the heart to be seen. Sometimes an iodine-based dye (contrast dye) is injected into one of veins during the scan, which highlights the coronary arteries on the X-rays. This type of CT scan is called a coronary CT angiography (CTA). Digital geometry processing or mesh processing is the method and area of research used to reconstruct, analyze, manipulate, simulate, and transmit a complex 3D model for volumetric (3D) representations of structures.

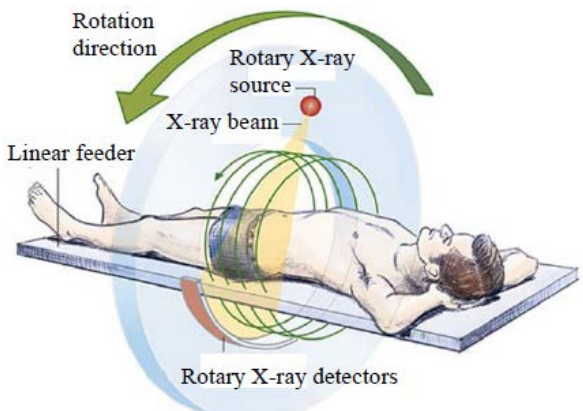

Fig.6. A CT scanning mechanism.

Many CT systems today perform "spiral" scanning and are capable of obtaining multiple slices simultaneously (using multiple X-ray tubes and channels, such as 64- and 764-channel CCT systems), with a substantial increase in spatial and temporal resolution and reduce artifacts. This allows relatively larger volumes of anatomy (e.g., 320 slices per rotation) to be imaged in relatively less time $(<1$ second per rotation) [26]; the entire heart volume can be scanned within a single breath hold (15-20 s) or shorter. This advancement has made it possible to visualize the beating heart, which has greatly increases CCT's popularity for detecting and quantifying CAD. By far, CCT is the fastest-growing noninvasive diagnostic cardiac imaging modality in the United States [27]-[29]. Another technology - electron beam CT (EBCT) - does not require any moving parts to generate the individual slice. As a result, the EBCT scanner allows a quicker image acquisition than conventional CT scanners, although the principle of creating cross-sectional images is the same as the conventional CT, whether single- or multi-slice scan. Future efforts on improvement of CCTs include increasing scan channels, reducing contrast injection volumes, removing artifacts related to patient breath-hold compliance.

One of the most unique applications of CCT is coronary angiography. CCT can easily determine the 3D relationship of anomalous coronary arteries with the aorta and pulmonary arterial trunk and detect aneurysms on coronary vessels, arteriovenous fistulae, and myocardial bridges. With the use of pulmonary vein isolation procedures for atrial fibrillation, CCT allows evaluation of the left atrial and pulmonary vein anatomy; establishment of the anatomic position of the esophagus to avoid its perforation during the procedure; and detection of pulmonary vein stenosis after the procedure [30]. High image quality of CT allows accurately diagnose certain HDs that other technologies cannot or have technical limitations to detect, such as: chest pain (ECG cannot be interpreted), pulmonary vein isolation, complex congenital 
heart disease, indeterminate stress test results, valvular disease (native or prosthetic valves), or coronary stents.

The effective dose of radiation, in millisieverts (mSv), depends on the volume of acquisition, the duration of the scan, and the radiation energy level used. The radiation energy level required to obtain adequate image quality depends on the width and thickness of the patient's chest, the object to be inspected, and the desired spatial resolution. Current CCT systems with 64 detectors provide a typical dose range on the order of 8-20 mSv for coronary imaging; 2-6 mSv for invasive imaging; and $10-27 \mathrm{mSv}$ for rest-stress nuclear myocardial perfusion imaging. One way to minimize the effective dose in CT is dose modulation which, depending on heart rate, may reduce total radiation exposure up to $50 \%$. Another strategy developed by some manufacturers involves step-and-shoot acquisition in a nonhelical mode that can reduce the dose to $2-4 \mathrm{mSv}$.

\section{Magnetic Resonance IMAging (MRI)/NuCLEAR MAGNETIC RESONANCE IMAGING (NMRI)}

Cardiac Magnetic Resonance Imaging (MRI) or Nuclear Magnetic Resonance Imaging (NMRI) uses radio waves (typically $10 \mathrm{M}-100 \mathrm{MHz}$ ), a static magnet field, detecting coils, and a computer to create pictures of the heart or blood vessels. First, the patient is placed in a very powerful magnetic field of 0.3 to 3 Tesla (specialized MRIs up to 7 T). This strong field then causes hydrogen atoms (H-atoms) within the organs (usually from water in the tissue and blood) to be magnetized and align themselves parallel with the magnetic field, either in the same direction or opposite to the direction of the field. To create a "slice", a short and powerful radio signal is sent through the body, perpendicular to the main magnetic field, causing the $\mathrm{H}$-atoms to become excited (have a higher energy level) and start to resonate with the frequency of the exciting wave. When the radio signal is turned off, the hydrogen atoms will, after a period of time, return to their original energy state. The excitation energy, which they had gained, is now released in the form of radio waves, which are detected by RF coil sensors (antennas). The strength of this signal is proportional to the proton density. The time it takes for the excited $\mathrm{H}$-atoms to return to their original energy level, the relaxation time, can also be measured and analyzed by a computer. Either the density, or the relaxation time, or their combination can be used for imaging [31]. The position is provided by the $\mathrm{x}, \mathrm{y}$ and $\mathrm{z}$ gradient coils. The collected data is reconstructed into a two-dimensional illustration through any axis of the body. Bones are virtually void of water and therefore do not generate any image data. This leaves a black area in the images. Thus, MRI is best suited for imaging soft tissue. MRI can produce both still and moving images of the heart and major blood vessels and measure blood flow in the heart. MRI is feasible to measure heart rate (HR) and heart rate variability (HRV) together with mechanical movement of the heart.

One of research on magnetic detectors for MRI devices is to use superconducting quantum interference detectors (SQUIDs). Using SQUID detectors can eliminate the needs for (1) a large, expensive, superconducting magnet as the imaging can be performed at very low magnetic fields; (2) the extensive magnetic shielding since SQUID gradiometers are sensitive to only the local change in magnetic field rather than the overall field; (3) the big, strict electrical shielding. Thus, only a minimal electrical shielding is required and it can be made small and lightweight [32]. In addition, the efficiency of the magnetic pickup is independent of the radio frequency and little radio frequency power is required to operate. Other research on magnetic detectors in NMR applications includes developing atomic magnetometers [33] with sensitivity potentially greater than that of SQUIDs and portable MRI instruments.

MRI is considered widely to be the most sensitive, most accurate and safest modality available for use in studying the internal soft tissues of the human body. One of the greatest advantages of MRI is the ability to change the contrast of the images. Small changes in radio waves and magnetic fields can completely change the contrast of the image. Different contrast settings will highlight different types of tissue. Another advantage of MRI is the ability to change the imaging plane without moving the patient. Most MRI machines can produce images in any plane. In contrast to CT, MRI uses non-ionizing RF signals to acquire its images and is best suited for non-calcified tissue. It has no known side effects related to radiation exposure, and it is considered to be safer than CTs. However, CT usually is more widely available, faster, less expensive, and can be used on patients that have internal metallic implants (e.g., pacemakers).

\section{OTHER TECHNOLOGIES FOR CVD DETECTION}

Several other technologies are also available for CVD detection. Microwave imaging (MWI) or microwave tomography (MWT) uses the scattering phenomena of microwaves $(4-8 \mathrm{GHz})$ to image the heart and blood vessels. A transmitter radiates microwaves into the heart, while a number of antennae placed around the heart receive the scattered waves. Microwave imaging is non-ionizing radiation which is safer than ionizing radiation (i.e., X-rays). The low illumination power levels used in microwave imaging also make regular screening possible. MWT is low cost and much cheaper than CT and MRI.

Single Photon Emission Computed Tomography (SPECT) and Positron Emission Tomography (PET) are valuable molecular imaging technologies as both are capable of detecting minute amount of radioactive tracers. SPECT scanning of heart is the state-of-the-art, noninvasive nuclear imaging method. It uses radioactive tracers injected into the bloodstream. A special "gamma" sensor or camera picks up signals from the tracers as they move around the patient's chest, and then the signals are converted into the image of the heart by a computer. Doctors use SPECT to diagnose coronary or artery disease and determine if a heart attack has occurred, how well blood is flowing to the heart at rest and during exercise (called a nuclear stress test), and how well the heart is working. If one cannot exercise, he/she will receive a medicine to increase the blood flow in the heart as if he/she is exercising (called a chemical or pharmacologic stress) [34]. Critical effort for the future success of SPECT and PET technologies is to design new and specific tracers for 
detection, localization, and staging of a disease, which will require various sensors.

The combination of the above detection methods, such as blood pressure, heart sound (HS), ECG, ICG, and PPG analyses, provides complementary information on the heart function and the vascular status. It has been shown that ECG and ICG can be reliably measured with functional textile sensors; PPG acquisition has already been implemented in congestive heart failure (CHF) disease management systems. More and more research on CVD diagnoses focus on wireless sensing and communication, wireless network, wearable devices with information technologies. Kappiarukudil and Ramesh reported real-time monitoring and detection of heart attack using wireless sensor network and a dynamic data collection algorithm to collect the ECG signals at regular intervals according to the health risk perceived in each patient [35]. Cheng used a smartphone to detect irregular rhythmic beating of the heart and indicate the risks of heart diseases in real-time [36]. Portable heart sensing products have also been developed in recent years. Alive Technologies has developed a small size and long battery life (48 hours) portable heart monitoring device that uses Bluetooth and a 3-axis accelerometer [37]. Vivometrics developed LifeShirt which can be worn by patients for heart state and body state monitoring [38]. Equivital developed heart state and health state monitoring systems for people working in challenging and dangerous environments and for clinical trials [39].

\section{Conclusion}

This paper overviews sensor technologies used for heart disease diagnosis and research. The paper starts with the types of cardiovascular diseases, the earliest CVD diagnosis method-Traditional Chinese Pulse Diagnosis (TCPD) and current development for automatic TCPDs. Then ECG, ICG, and PPG waveforms and detecting methods are discussed. ECG features conductive sensing that directly uses electrodes for measurement. ICG is an impedance method that requires an AC current flow through the thorax in order to measure the impedance that relates to thoracic fluid levels to assess the cardiovascular status. With each cardiac cycle, the thoracic fluid levels change, causing the impedance change. A PPG is typically obtained using an optical method - a LED illuminates the skin on the finger and the amount of light either reflected or transmitted through the finger muscle is measured by a photo-detector. Thus the pulsatile changes of blood volume in tissue are converted to changes in light absorption. Echocardiograph (ultrasound) utilizes a piezoelectric element to generate a sound wave, and then pick up the echo by the same or a different sensor. Ultrasound sensors can create a moving picture of the heart or vessels. Acoustic sensors detect the sounds from the heart or blood flow in arteries and veins. Acoustic transducers used in CVD detection include piezoelectrical crystals, electromagnetic diaphragms, and capacitors. Cardiac CT uses X-ray to generate 2D sectional images. These 2D images are then packed together to form a 3D images. It can help to detect chest pain, pulmonary vein isolation, complex congenital heart disease, valvular disease, or coronary stents. Cardiac MRI uses $10-100 \mathrm{MHz}$ radio waves, a static magnet field, detecting coils, and a computer to create pictures of the heart or blood vessels. MRI is particularly useful for imaging soft tissue, and can produce both still and moving images of the heart and major blood vessels. The sensor technologies and development for CVD studies and diagnosis mainly focus on (1) detect heart pulse waveforms (e.g., ECG, TCPD); (2) measure blood properties (e.g., pressure, viscosity, flowrate, contents and their percentage); (3) create heart or vessel images (e.g., CT, MRI, Ultrasonic); and (4) establish wireless sensor network.

\section{REFERENCES}

[1] M. Naghavi, C. J. L. Murray, and A. Lopez, "Global, regional, and national age-sex specific all-cause and cause-specific mortality for 240 causes of death, 1990-2013: A systematic analysis for the global burden of disease study,” The Lancet, vol. 385, no. 9963, pp. 117-171, 2013

[2] X. Liu et al., "A new method of modeling of inquiry diagnosis for coronary heart disease in traditional Chinese medicine," in Proc. 4th Intl. Conf. on Biomedical Engr. and Informatics (BMEI), pp. 1631-1634, 2011.

[3] A. Zhang and F. Yang, "Study on recognition of sub-health from pulse signal,” in Proc. ICNNB'05, Intl. Conf. on Neural Networks and Brain. Beijing, China, 2005, pp. 1516-1518.

[4] Flaws, The Secret of Chinese Pulse Diagnosis, 2nd ed. Boulder, CO: Blue Poppy Press, March 1997.

[5] G. Maciocia and S. Ming, Pulse Diagnosis in the Foundations of Chinese Medicine, China: Elsevier, 2005.

[6] E. Lau and A. Wang, "Relationship between wrist-pulse characteristics and body conditions," in Proc. EM'00, 14th Engr Mechanics Conf. of the American Society of Civil Engineers, 2000, Austin, USA.

[7] S. Walsh and E. King, Pulse Diagnosis: A Clinical Guide, 1st ed. New York, Churchill Livingstone, Dec. 2007.

[8] Q. Wu, "Power spectral analysis of wrist pulse signal in evaluating adult age,” in Proc. 2010 Intl Symposium on Intelligence Information Processing and Trusted Computing, pp. 48-50, 2010.

[9] S. Nair and W. Du, "Development of an automatic traditional Chinese medicine pulse meter,” 2010 Research Report, San Jose State University.

[10] C. Luo et al., "Possibility of quantifying TCM finger-reading sensation: I. bi-sensing pulse diagnosis instrument,” European J. of Integrative Medicine, vol. 4, no. 3, pp. 255-262, 2012.

[11] Y. Chu et al., "A new pulse pillow of traditional Chinese medicine The wrist fixer system," in Proc. 2013 International Conference on Orange Technologies (ICOT), 2013, pp. 11-14.

[12] J. Peng and S. Lu, “A flexible capacitive tactile sensor array with CMOS readout circuits for pulse diagnosis,” IEEE Sensors J., vol. 15, no. 2, pp.1170-1177, 2015.

[13] B. Wang and J. Xiang, "Detecting system and power-spectral analysis of pulse signals of human body,” in Proc. ICSP'98, 4th Inter. Conf. on Signal Processing, 1998, pp.1646-1649, Beijing, China.

[14] W. Du and W. Jose, "Design of a modular signal conditioning circuit for biopotential sensors," Sensors \& Transducers Journal, vol. 120, no. 9, pp. 1-12, September 2010.

[15] J. Luprano et al., "HeartCycle: Advanced sensors for telehealth applications,” in Proc. Engr in Medicine and Biology Society (EMBC), 35th Annual Intl. Conf. of the IEEE, 2013, pp. 6984-6987.

[16] "ICG impedance cardiograph, non-invasive hemodynamic measurements,” Philips, Oct. 2004.

[17] X. Chen et al., "Noninvasive ambulatory monitoring of electric-mechanical function of heart with a multifunction wearable sensor,” in Proc. IEEE 38th Annual Intl. Computers, Software \& Applications Workshops, 2014, pp. 662-667.

[18] K. Shelley and S. Shelley, "Pulse oximeter waveform: Photoelectric plethysmography," in Clinical Monitoring, Carol Lake, R. Hines, and C. Blitt, Ed. W.B. Saunders Company, 2001, pp. 420-428.

[19] U. Timm et al., "Optical sensor system for non-invasive blood diagnosis,” in Proc. IEEE Sensors Applications Symposium, 2009, pp. 240-244.

[20] T. Lo, T. Escorcio, and R. J. Chang, "Ultrasonic monitor for measuring heart and pulse rates,” US Pat. Nos. 6,371,920 B1, 2004.

[21] P. Kshirsagar. (November 2013). How Indian technology is fighting heart disease. ARTSENS. [Online]. Available: 
http://www.firstpost.com/living/artsens-the-indigenous-technology-th at-can-fight-heart-disease-1216593.html

[22] H. J. Freeman and J. M. Dinwiddie, "Cardiac monitor wristwatch,” US Pat. No. 4,086,916, 1975.

[23] K. Tsubata, "Device for detecting pulse waves,” US Pat. No. 6,447,456 B1, 2002.

[24] B. M. Duff, C. S. Gibson, D. C. Winter, and H. H. Peel, "Method an apparatus for detection of heart disease,” US Patent No.: 4905706 A 1988.

[25] Computed tomography. (May 2015). [Onine]. Available: www.iambiomed.com/equipments/ct

[26] T. G. Flohr et al, "First performance evaluation of a dual-source CT (DSCT) system,” Eur Radiol, vol. 16, no. 2, pp. 256-268, 2006.

[27] M. J. Garcia, "Noninvasive coronary angiography: Hype or new paradigm?” JAMA, vol. 293, no. 20, pp. 2531-2533, 2005.

[28] P. Schoenhagen et al., "Non-invasive coronary angiography with multi-detector computed tomography: Comparison to conventional x-ray angiography,” Intl. J. Cardiovascular Imaging, vol. 21, no. 1, pp. 63-72, 2005.

[29] M. H. Hoffmann et al., "Noninvasive coronary angiography with 16-detector row CT: Effect of heart rate,” Radiology, vol. 234, no. 1, pp 86-97, 2005.

[30] S. Prat-Gonzalez, J. Sanz, and M. J. Garcia, “Cardiac CT: Indications and Limitations,” J. Nucl. Med. Technol., vol. 36, no. 1, 18-24, March 2008.

[31] Medfysica. (May 2015). [Online]. Available: http://onderwijs1.amc.nl/medfysica/doc/MRI_general.htm

[32] M. Cohen, "Portable device for ultra-low field magnetic resonance imaging (ULF-MRI)," Patent Pub. No.: WO2008008447 A2, 2008.

[33] H. Savukov, "Magnetic sensing method, atomic magnetometer and magnetic resonance imaging apparatus,” Patent No. WO2008149957 A2, 2008.

[34] American Heart Association Handouts, May 15, 2015.
[35] K. Kappiarukudil and M. Ramesh, "Real-time monitoring and detection of "heart attack" using wireless sensor networks," in Proc. 2010 Fourth International Conference on Sensor Technologies and Applications (SENSORCOMM), 2010, pp. 632-636.

[36] A. C. Cheng. Real-time cardiovascular diseases detection on a smartphone. [Online]. Available: http://research.microsoft.com/en-us/events/mhealth2009/cheng-poster. pdf

[37] Alive Technologies. (May 2015). [Online]. Available: www.alivetec.com

[38] Vivonoetics. (May 2015). The lifeshirt system, manufactured by VivoMetrics is a comfortable, lightweight garment with embedded sensors that continuously collects information on a range of cardiopulmonary parameters. [Online]. Available: http://vivonoetics.com/products/sensors/lifeshirt/

[39] Equivital. (May 2015). [Online]. Available: www.equivital.co.uk

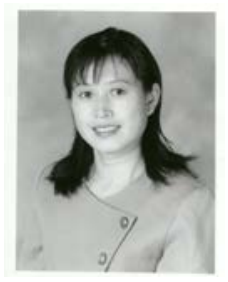

Winncy Du earned her $\mathrm{PhD}$ and her MS degree in 1999 from Georgia Technology. She earned first MS and BS degrees from West Virginia University in 1994 and Jilin University in 1983, respectively.

She is the director of the Robotics \& Sensor Laboratory; a sole author of book: Resistive, Capacitive, Inductive, and Magnetic Sensor Technologies (Taylor \& Francis Group, 2015), a contributor to the books: Smart Sensors and Sensing Technology (Springe-Verlag, 2008) and Modern Sensors, Transducers, and Sensor Networks (IFSA, 2012). She is the fellow of ASME. Her professional honors include the International ASME Diversity \& Outreach Award, ASME District D Student Section Advisor Award, Richard A. Fitz Outstanding Faculty Advisor Award, Faculty Award for Excellence in Scholarship and Newman Brothers Award for Faculty Excellence at SJSU. 